\title{
ENERGY EFFICIENT MULTI HOP CROSS LAYER DESIGN FOR LARGE SCALE WIRELESS SENSOR NETWORKS
}

\author{
Bharti Goyal $^{1}$, Kanika Sharma ${ }^{2}$
}

\begin{abstract}
With the fast development of communication system and to have the continued life-time, Energy adequacy is the central concern in this network. Consequently, to boost network lifetime and to attain topmost reliability and scalability, the routing techniques have been refined. (LEACH) Low Energy Adaptive Clustering Hierarchy is energy adequate hierarchical, clustering based protocol, which is supposed as the center of various clustering hierarchical protocols. Therefore, the investigation in this thesis will be oriented towards few recently tailored protocols that will put forward toempower the efficiency of LEACH protocol. In this paper, the Cluster Head selection is done by incorporating distance factor along with Energy and SNR. Moreover, the inter cluster communication is handled by incorporating distance factor so as to minimize energy consumption in the network. It is observed that value of FND, HND and LND for MHLBP is 2080, 3205 and 3595 rounds respectively. Again value of evaluating parameters FND, HND and LND for proposed network is 2968,3416 and 4442 rounds respectively. Thus for FND result is improved by $42.69 \%$, for HND result is improved by $56.58 \%$ and for LND is improved by $23.56 \%$.
\end{abstract}

\section{INTRODUCTION}

Remote sensor systems (WSN) should be the one of most extreme vivacious advances now days. In the development of Micro-electronic-mechanical-frameworks (MEMS) has awfully influenced the extension of packed sensor hubs [1]. The smaller than expected hubs collaborate with each together by means of RF correspondence in SMI (Scientific, Medical and Industrial) band to make Wireless Sensor Network. Remote Sensor Networks comprise of scaled down hubs that are contained in with sensors, microchip, memory, handset, and voltage supply. The savvy sensor hubs are spread out in a particular field and with help of the remote connections they are organized, that yield fabulous extensions for various applications. In a WSN the information data that is gathered by the hubs alluded as sink [2]. The sink might cooperate through an association with the outside world utilizing web where inside time restricts the data can be used.

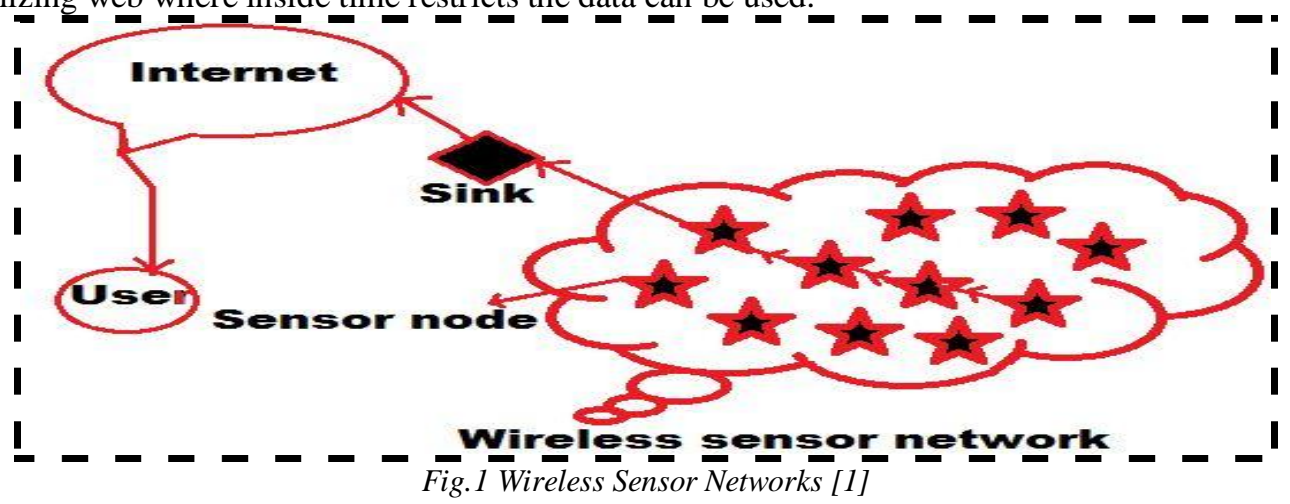

The principle point of WSNs is as per the following:

a) To compute the estimation of physical factors at a known position.

b) To discover the occurrence of the occasions which are of concern and approximated element of the spotted episode.

c) Briefly group a spotted episode and following an occurrence to happen.

\section{System Architecture}

A sensor node is consists of four main units as shown in Fig.2

1. Sensing unit

2. Processing unit

\footnotetext{
${ }^{1}$ M.E. Student, Department of ECE, NITTTR, Chandigarh

${ }^{2}$ Assistant Professor, Department of ECE, NITTTR, Chandigarh
} 
3. Transceiver unit

4. Power unit

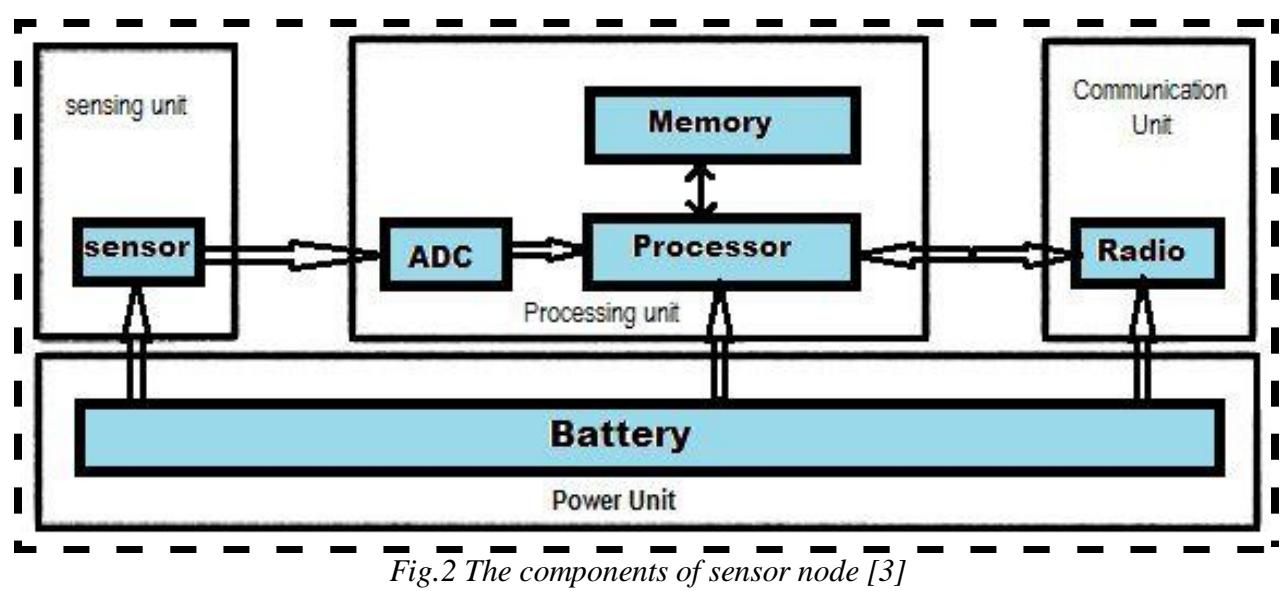

They may also have application dependent additional components such as a location finding system, a power generator and a mobilizer.

I. There are two further parts of sensing units:

i. Sensors

ii. Analog to Digital Converters (ADCs):- the digital signals by the ADC is received after getting converted from the analog signals produced by the sensors based on the observed phenomenon and then delivered into

II. Related Work: the processing unit.

Yun Li et.al. in [4] proposed the technique to set the time length of each round, to increase throughput and prolong the lifetime of the network, which is indicated as the amount of data packets sent to the sink node. HamedJelodar et.al. in [5] discussed various methods to reduce the enrgy consumption so that network lifetime can be improved further. In this paper two particular algorithms LEACH and HEED are investigated. Hence, concluded that cluster head play an important role in management of energy consumption.Shoushi et.al. in [6] presents an energy-efficiency Optimized LEACH-C. First cluster head is selected with energy more than the average then quadratic sum of distance from each $\mathrm{CH}$ to its member nodes to get optimal solution. Then for next round each node with residual energy greater than the estimated is taken to get better solution. Hence, the loss of $\mathrm{CH}$ for each round is minimized, and WSN lifetime is extended ultimately.RajuDuttaet.al.in [7] describes the network quality that depends on different characteristics of data transmission as a Modification on LEACH protocol and explained the comparison of magnitude, phase, phase delay, group delay, amplitude of broadcasting and energy consumption respectively. JunyoungHeoet.al.in [8] proposed the concept of ERAQ. In EARQ, a node estimates the energy cost, delay and reliability of a path to the sink node, based only on information fromneighboring nodes. Then, it calculates the probability of selecting a path, using the estimates. Chi-Tsun Cheng et.al.in [9] proposed a delay-aware data collection network structure and its formation algorithms. To cater for different applications, network formation can be implemented in either centralized or decentralized manner. Yanjun Yaoet.al. in [10] developed one data collection protocol called EDAL, which stands for Energy-efficient Delay-aware Lifetime-balancing data collection which is inspired by recent techniques developed for open vehicle routing problems with time deadlines (OVRP-TD) in operational research. S. K. Singh et.al.in [11] surveyed the variants of LEACH routing protocols proposed so far and discussed the enhancement and working of them. In this survey classification of all the protocols in two sections, namely, single hop communication and multi-hop communication based on data transmission from the cluster head to the base station is done. Jianhua Huang et.al.in [12] proposed an energy-efficient multi-hop routing protocol based on grid clustering to prolong the network lifetime. In this the network area is divided into uneven grids to form clusters with differentLevels based on the distance from the cluster head. Then multi hop technique is used within the cluster.

\section{Problem Definition}

As we know the Wireless sensor network consist of the sensor nodes that are mechanically turned on through battery, to interact with one another for environment supervising. On account of the supervision nodes are spread out in either way over a vast area but frequent change or restoration of battery is not practicable for some applications based on this network.

Hence, to have continued life-time, Energy adequacy is the central concern in this network and will focus on to improve its energy efficiency further and to prolong the lifetime so that it can be used for long period of time.

\section{The Proposed Protocol}


The proposed protocol aims to enhance the network lifetime. It follows these steps to implement the proposed technique of incorporating the two cluster heads in a cluster.

a) Initialization of parameters

b) Network Formation

c) Simulated Results

a) Initialization of Parameters: The very first step while simulating the proposed technique is to initialize the simulation parameters that defines the size of network, number of nodes, energy of nodes that are to be deployed along with the energy requirement for the aggregation of data and transmission and reception of data

b) Network Formation

After initialization of simulation parameters, the sink is deployed for the two network scenario as mentioned above. Fig. 3 shows the MATLAB generated randomly deployed 200 sensor nodes in $200 \mathrm{~m} \mathrm{X} \mathrm{200m} \mathrm{network} \mathrm{area} \mathrm{and} \mathrm{the}$ position of sink node is $(100,200)$. Initially all nodes contain same energy which is $0.5 \mathrm{~J}$.All sensor nodes and sink node are static.

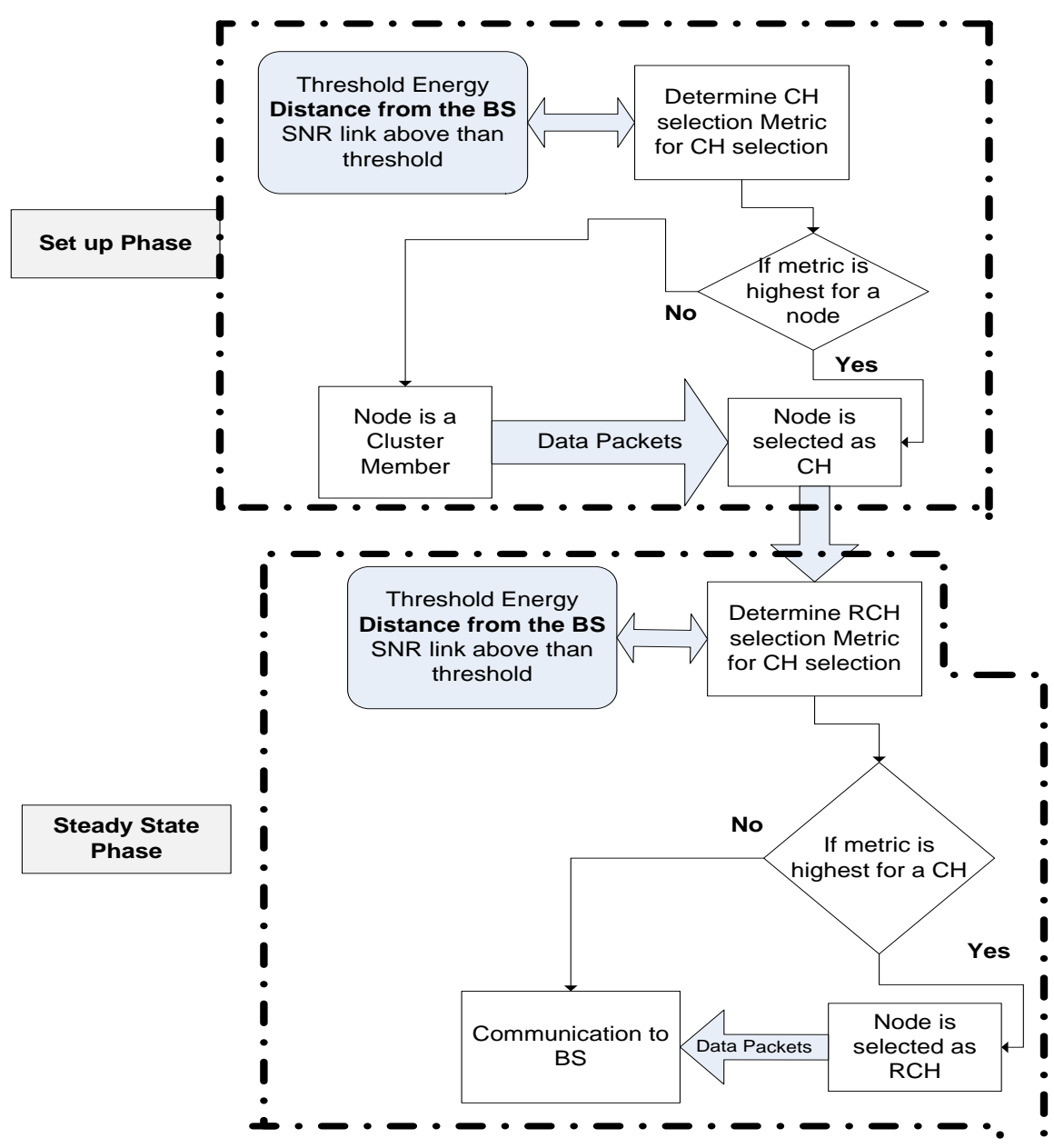

Table 1 Simulation parameters

Fig. 3Flow Diagram for setup phase and steady state phase for proposed work

\begin{tabular}{|ll|}
\hline Parameter & Value \\
\hline Area of network & $200 \mathrm{~m} \mathrm{X} \mathrm{200m,}$ \\
\hline Packet size & $800 \mathrm{bits}$ \\
\hline Number of nodes & 200 \\
\hline Position of sink & $(100 \mathrm{~m} \mathrm{X} \mathrm{200m)}$ \\
\hline Initial energy & $0.5 \mathrm{~J}$ \\
\hline $\mathbf{E}_{\text {elec }}$ & $50 \mathrm{~nJ} / \mathrm{bit}$ \\
\hline $\mathbf{E}_{\mathbf{R x} \text {-elec }}$ & $50 \mathrm{~nJ} / \mathrm{bit}$ \\
\hline $\mathbf{E}_{\text {amp }}$ & $0.0013 \mathrm{pJ} / \mathrm{bit} / \mathrm{m} 2$ \\
\hline
\end{tabular}




\begin{tabular}{|ll|}
\hline Transmission Range & $100 \mathrm{~m}$ \\
\hline Desired \% of cluster head & $5 \%$ \\
\hline Energy for data fusing & $5 \mathrm{~nJ} / \mathrm{bit}$ \\
\hline
\end{tabular}

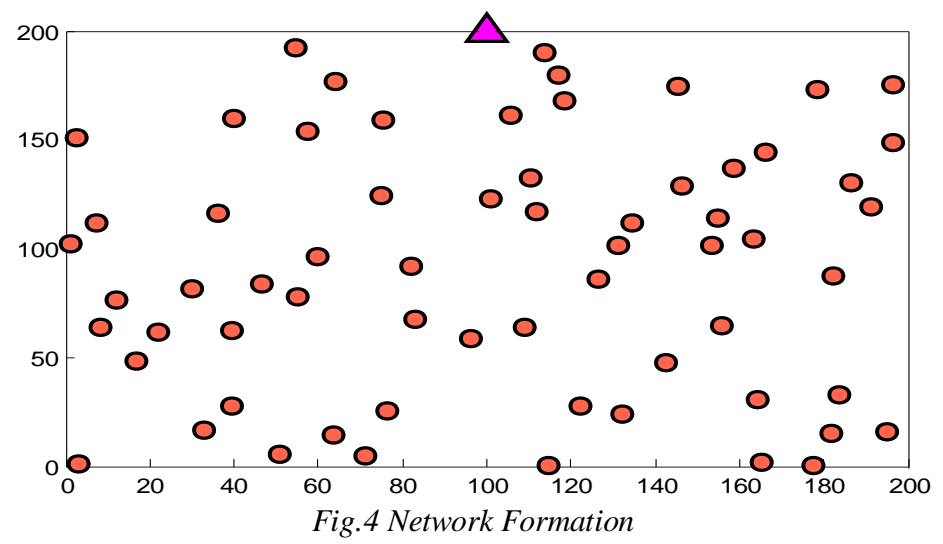

\section{c) Simulation Results}

The proposed work is simulated in MATLAB 2013a. The proposed protocol is implemented for multi hop routing. The protocol works for the cluster head selection by using distance approach along with the energy only. Inter cluster communication is made effective by introducing the distance factor and energy factor in the routing process. The simulated results of proposed algorithm and MHLBP (multihop LEACH based protocol) are compared in this section.

\section{i. No. of dead nodes v/s No. of rounds}

Fig. 5 shows the simulation graph of no. of dead nodes v/s no. of rounds for 200 nodes. Graph gives an idea about the number of rounds at which the number of nodes becomes dead.

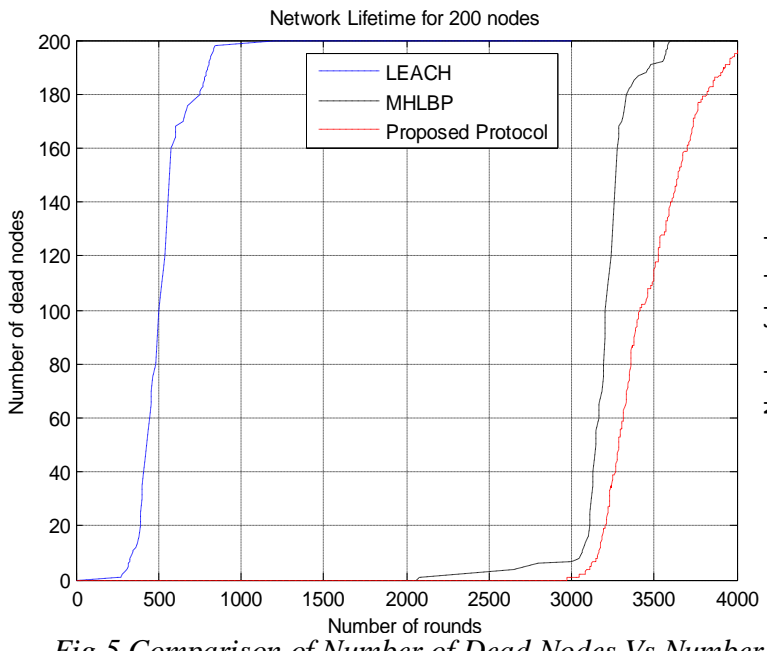

Fig. 5 Comparison of Number of Dead Nodes Vs Number of Rounds for 200 nodes

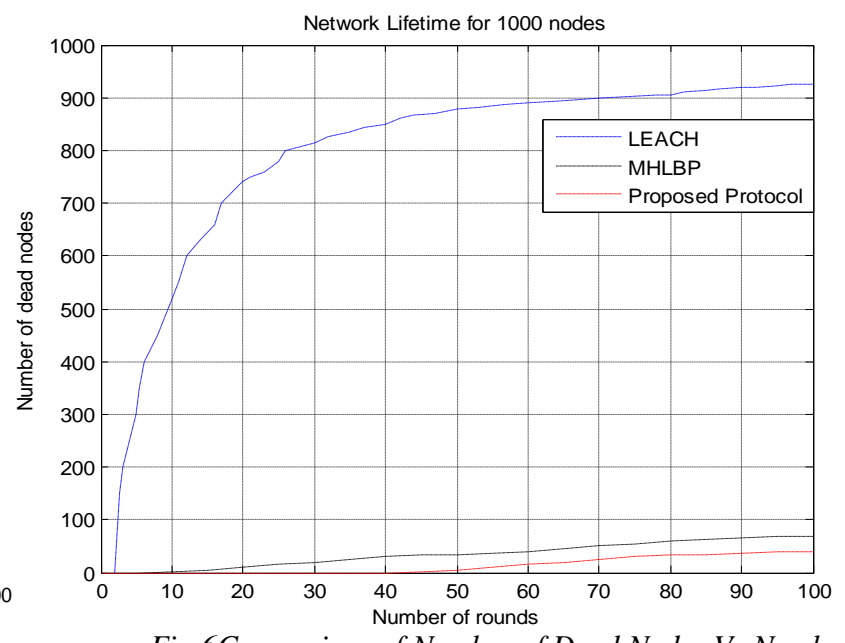

Fig.6Comparison of Number of Dead Nodes Vs Number of Rounds for 1000 nodes

Fig. 6 shows the simulation graph of no. of dead nodes $v / s$ no. of rounds for 200 nodes. Graph gives an idea about the number of rounds at which the number of nodes becomes dead.

\section{ii. Average residual energy v/s No. of rounds:}

Fig. 7 shows the simulation graph of Average residual energy v/s no. of rounds for 200 nodes. Graph gives an idea about the comparison of residual energy of the proposed network with the existing one and hence, proved that the proposed algorithm is more energy efficient than the MHLBP (Multi hop LEACH based protocol) for the given number of nodes. 

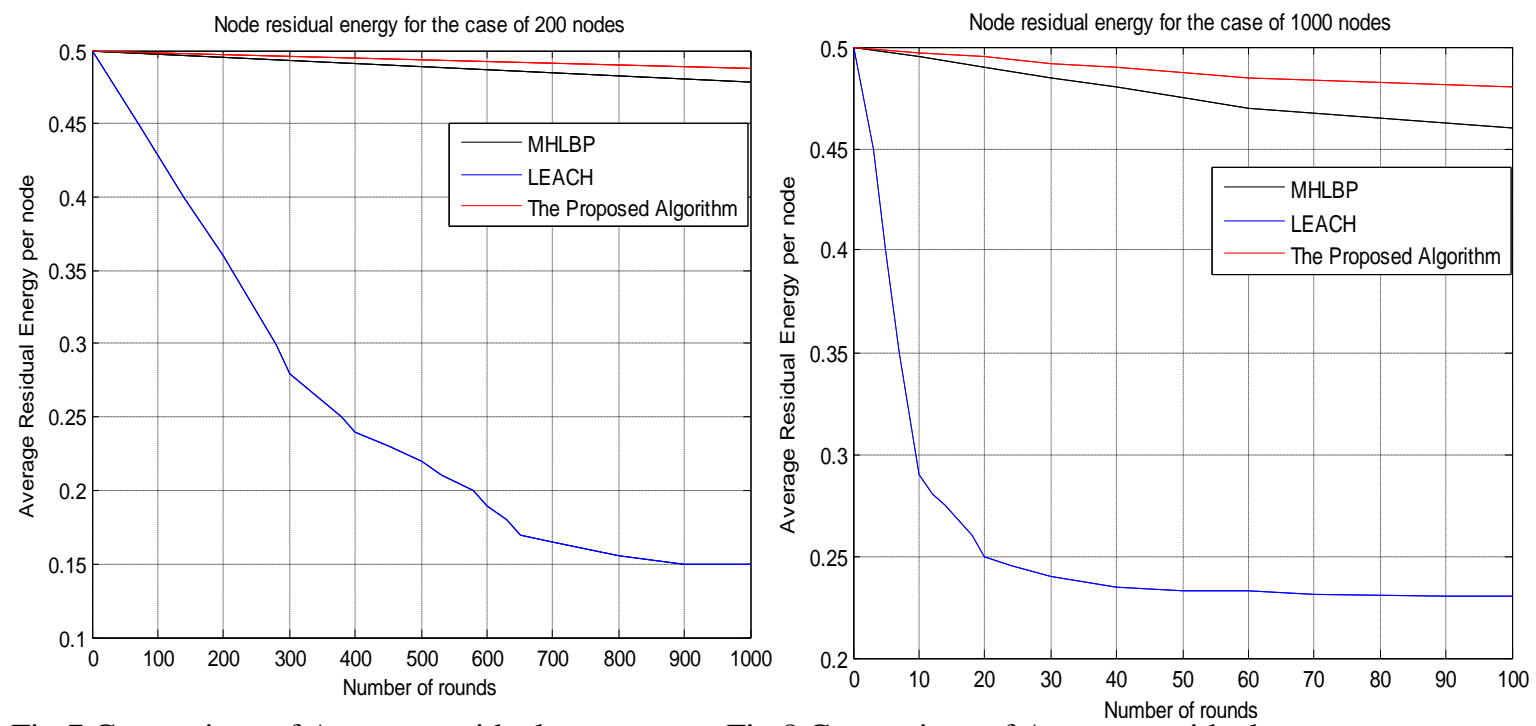

Fig.7 Comparison of Average residual energy

Fig.8 Comparison of Average residual energy

Vs Number of Rounds for 200 nodes

Vs Number of Rounds for 1000 nodes

Fig. 8 shows the simulation graph of Average residual energy v/s no. of rounds for 200 nodes. Graph gives an idea about the comparison of residual energy of the proposed network with the existing one and hence, proved that the proposed algorithm is more energy efficient than the MH-LEACH for the given number of nodes.

iii. Packets delivery Ratio v/s Packets arrival rate: Figure 9 shows the comparison of Packets delivery ratio to the packets arrival rate of the proposed algorithm with the existing MHLBP (Multi hop LEACH based protocol). And showed that packet delivery ratio of the proposed network is improved than the existing one.

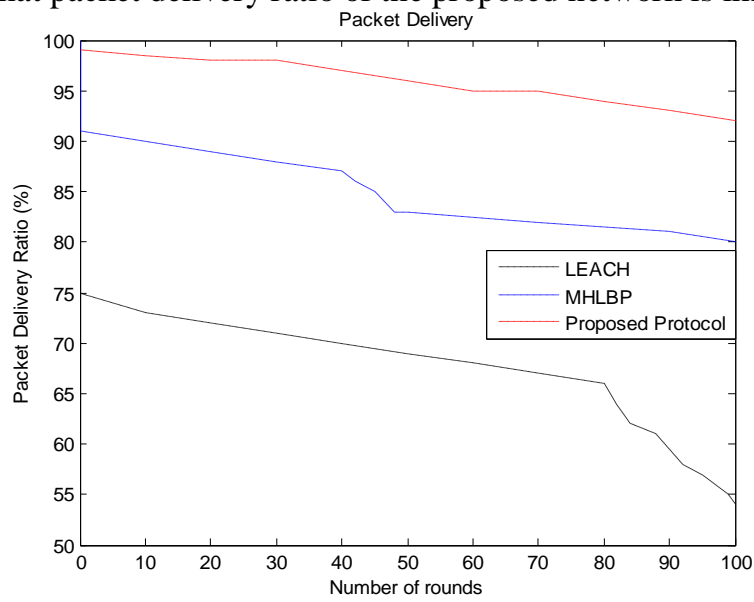

Fig.9 Packets delivery ratio with 200 nodes

iv. Comparison of FND, HND and LND: FND indicates the number of rounds when first sensor node dies. HND indicates the number of rounds when half sensor node dies and LND indicates the number of rounds when last sensor node dies.

Table2 comparison of algorithm based on FND, HND and LND

\begin{tabular}{|l|l|l|l|}
\hline Algorithm & LEACH & MHLBP & PROPOSED ALGORITHM \\
\hline FND (rounds) & 250 & 2080 & 2968 \\
\hline HND (rounds) & 500 & 3205 & 3416 \\
\hline LND (rounds) & 1200 & 3595 & 4442 \\
\hline
\end{tabular}

Table 2 shows that FND for LEACH and MHLBP are 250 rounds and 1374 rounds respectively. Means for LEACH first sensor node dead on 250 rounds and for MHLBP it is 2080 rounds but in case of proposed algorithm first sensor 
node becomes dead on 2968 rounds. Similarly HND for LEACH, MHLBP and proposed algorithm are 500 rounds, 3205 rounds and 3416 rounds respectively. Again LND for LEACH, MHLBP and proposed algorithm are 1200 rounds, 3595 rounds and 4442 rounds respectively. This shows that simulation results are improved by $42.69 \%$, $56.58 \%$ and $23.56 \%$ for FND, HND and LND respectively if compared to MHLBP algorithm. Fig. 10, 11 and 12 shows the graphical comparison of FND, HND and LND for LEACH, MHLBP and proposed algorithm.

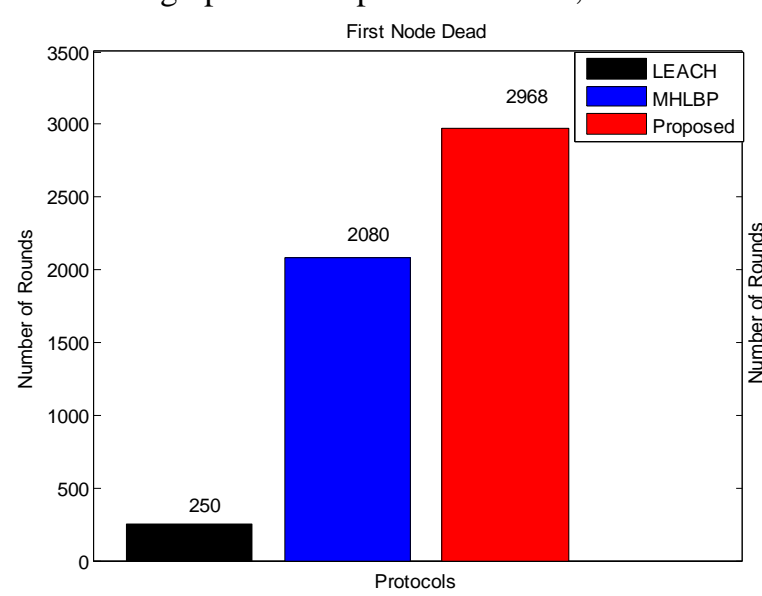

Fig.10 First node dead (FND)

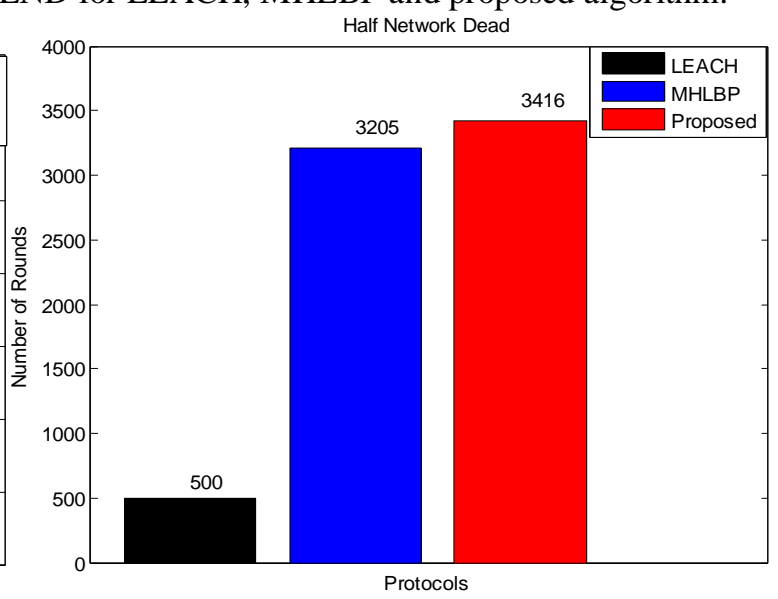

Fig. 11 Half node dead (HND)

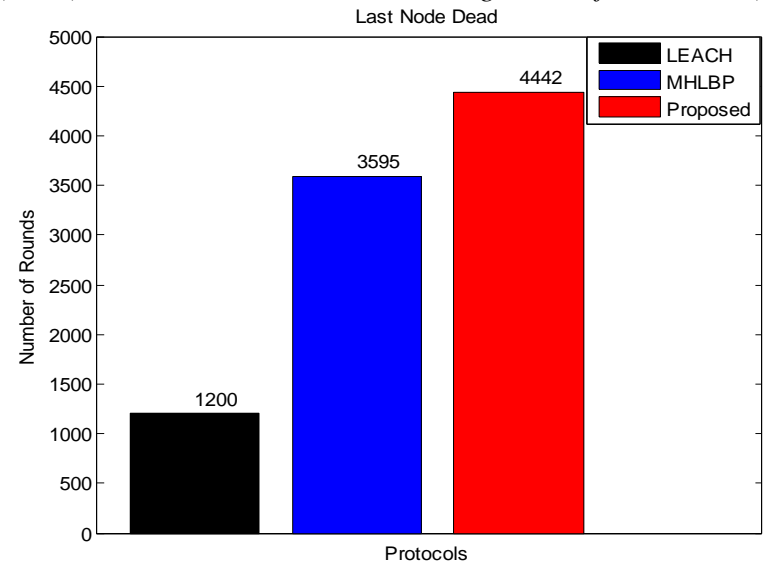

Fig. 12 Last node dead (LND)

\section{Conclusion}

Wireless Sensor Network has facilitated us with number of applications which are limited by human imagination. Routing in WSN is one of the most prominent areas of research which aims to make the sensor network energy efficient. With the development and advancement in the various routing protocols Multi hop made its huge impact on the energy efficient approach of WSN. Multi hop not only enhances the network lifetime but it also adds energy balancing in to the network. LEACH being the first protocol to exploit clustering by employing the single hop communication to the Base Station. Random selection of $\mathrm{CH}$ cost this network with a huge amount of energy consumption which results in expiry of sensor network. Value of FND, HND and LND for MHLBP is 2080, 3205 and 3595 rounds respectively. Again value of evaluating parameters FND, HND and LND for proposed network is 2968,3416 and 4442 rounds respectively. Thus for FND result is improved by $42.69 \%$, for HND result is improved by $56.58 \%$ and for LND is improved by $23.56 \%$.

\section{REFERENCES}

[1] KhushbooManohar, A.I. Darvadiya, "Study of Leach Protocol- A Review," International Journal of Modern Trends in Engineering and Research, pp. 401-407, 2014.

[2] SangitaVishwakarma, "An analysis of LEACH Protocol in Wireless Sensor Network: A Survey," International Journal of Computer Science \& Engineering Technology (IJCSET), Vol. 6, pp. 148-153, March 2015.

[3] AlakeshBraman, Umapathi G. R, "A Comparative Study on Advances In LEACH Routing Protocol for Wireless Sensor Networks: A survey," International Journal of Advanced Research in Computer and Communication Engineering, Vol. 3, No. 2, pp. 5683-5690, February 2014.

[4] Manpreetkaur, Surender Singh, "A Comparative Study on LEACH Routing Protocol and its Variants in Wireless Sensor Networks: A Survey,” IJIRT, Vol. 2, No. 1, pp. 113-119, June 2015. 
[5] AblolfazlAfsharzadehKazerooni, HamedJelodar, JavadAramideh, "Leach and Heed Clustering Algorithms In Wireless Sensor Networks: A Qualitative Study," in Advances in Science and Technology Research Journal, Vol. 9, No. 25, pp. 7-11, March 2015.

[6] Shuo Shi, Xinning Liu and XuemaiGu, "An energy-efficiency Optimized LEACH-C for wireless sensor networks," 7th International Conference on Communications and Networking in China, Kun Ming, pp. 487-492, August 2012.

[7] RajuDutta, Shishir Gupta and Mukul K Das, "Improvement on LEACH Protocol in Wireless Sensor Networks," in International Journal of Computer Applications (0975 - 8887), Vol. 97, No.21, pp. 36-40, July 2014.

[8] JunyoungHeo, Jiman Hong, and Yookun Cho, Member, "EARQ: Energy Aware Routing for Real-Time and Reliable Communication in Wireless Industrial Sensor Networks," IEEE Transactions on Industrial Informatics, Vol. 5, No. 1, pp. 3-11, February 2009.

[9] Chi-TsunCheng, Chi K. Tse, Francis C. M. Lau, “A Delay-Aware Data Collection Network Structure for Wireless Sensor Networks,” IEEE Sensors Journal, Vol. 11, No. 3, pp. 699-710, March 2011.

[10] Yanjun Yao, Qing Cao, Athanasios V. Vasilakos, "EDAL: An Energy-Efficient, Delay-Aware, and Lifetime-Balancing Data Collection Protocol for Heterogeneous Wireless Sensor Networks," IEEE/ACM Transactions On Networking, Vol. 23, No. 3, pp. 810-823, June 2015.

[11] S. K. Singh, P. Kumar and J. P. Singh, "A Survey on Successors of LEACH Protocol," in IEEE Access, Vol. 5, pp. 4298-4328, February 2017.

[12] Jianhua Huang, Yadong Hong, Ziming Zhao, Yubo Yuan, "An energy-efficient multi-hop routing protocol based on grid clustering for wireless sensor networks," in cluster computing (Springer), pp. 1-13, June 2017. 